\title{
Effect of Stress Echocardiography Testing on Changes in Cardiovascular Risk Behaviors in Postmenopausal Women: A Prospective Survey Study
}

\author{
Francesca Mantovani, MD, Sahar S. Abdelmoneim, MBBch, MSc, MS,1,3 Victoria Zysek, MD, \\ Susan Eifert-Rain, RN, and Sharon L. Mulvagh, $\mathrm{MD}^{1,2}$
}

\begin{abstract}
Background: We evaluated the impact of contrast stress echocardiography (CSE) testing results on cardiovascular $(\mathrm{CV})$ risk behaviors in postmenopausal women presenting with chest pain symptoms. This was a substudy of the Stress Echocardiography in Menopausal Women at Risk for Coronary Artery Disease (SMART) trial.

Methods: From 2004 to 2007, 366 women (mean age 54.4 5.5 years, range 40-65; body mass index (BMI) 31.4 \pm 6.68; Caucasian in 95\%) completed CSE and were invited to participate in the Women's Heart Clinic Risk Assessment Questionnaire (WHCRAQ survey) at the time of CSE and after 2 years. Of the 366, 203 (55\%) postmenopausal women completed both the baseline and 2-year follow-up surveys (age $61 \pm 5$ years; Framingham risk score $6 \pm 4 \%, 81 \%$ treadmill CSE, $19 \%$ dobutamine CSE). WHCRAQ assessed medical history, hormone therapy (HT), and CV risk behaviors (cigarette smoking history, including current smoking and mean cigarettes smoked per day; physical activity or exercise, including mean exercise minutes per week; and dietary fat intake, alcohol intake, and mean alcohol drinks per week). Abnormal CSE was defined as a new or worsening stress wall motion abnormality. Post-CSE changes in CV risk behaviors were determined by comparing baseline versus 2-year data.

Results: Of the 203 women who completed the survey at baseline and 2-year CSE, 29 were excluded to avoid confounding effect (coronary angiography [CA] was performed during follow-up). Of 174 women (55\% hypertensive, $10 \%$ diabetic, $76 \%$ hyperlipidemic, and $25 \%$ on HT), CSE was abnormal in $10 \%$. Baseline characteristics were not significantly different in normal versus abnormal CSE, apart from diabetes (8\% vs. $24 \%, p=0.04)$. Slightly more women with normal CSE were taking HT than not $(27 \%$ vs. $12 \%, p=0.19)$. CV risk behaviors that were different in normal versus abnormal CSE included current smoking, alcohol drinks per week, and higher fat diet (8\% vs. $24 \%$, $p=0.03 ; 2.5 \pm 3.5$ vs. $1.94 \pm 5.2, p=0.031$; and $92 \%$ vs. $76 \%, p=0.03$, respectively). Cigarette smoking decreased (mean difference of -1.5 cigarettes per day, $p=0.014$ ) in the abnormal-CSE group, whereas number of alcohol drinks per week increased (mean difference $+0.38, p=0.009$ ) in the normal-CSE group.
\end{abstract}

Conclusion: We observed an association of lifestyle changes with CSE test results in postmenopausal women

\section{Introduction}

$\mathbf{I}$ MPROVEMENT IN RECOGNITION AND AWARENESS of coronary artery disease (CAD) symptoms and presentation represents a critical strategy to reduce cardiovascular (CV) deaths among women. ${ }^{1}$ The diagnostic accuracy of stress testing in women has historically been associated with a di- minished sensitivity and specificity for detection of obstructive CAD. ${ }^{2}$ The American Heart Association (AHA) consensus statement recommends adding an imaging modality for the evaluation of intermediate-risk women who either have a history of an abnormal exercise stress test or an abnormal baseline electrocardiography (ECG) or require pharmacologic stress testing owing to an inability to adequately

\footnotetext{
${ }^{1}$ Cardiovascular Ultrasound Imaging and Hemodynamic Laboratory, Mayo Clinic, Rochester, Minnesota.

${ }^{2}$ Women's Heart Clinic, Mayo Clinic, Rochester, Minnesota.

${ }^{3}$ Division of Cardiovascular Diseases, Assiut University, Assiut, Egypt.
} 
exercise. $^{2}$ Imaging techniques performed during dynamic exercise or pharmacologic stress offer several advantages, including superior diagnostic performance and the ability to localize and quantify areas of ischemia. Both echocardiography and nuclear stress imaging using myocardial perfusion scintigraphy (MPI) have been widely used in both women and men; recently, magnetic resonance imaging (MRI) and positron emission tomography (PET) scanning have become additional imaging options. Advantages of stress echocardiography include lack of ionizing radiation, relative low cost, and immediacy of interpretation. ${ }^{2}$ Furthermore, the use of contrast stress echocardiography (CSE) has been shown to be a safe technique that increases the confidence of interpretation and accuracy of standard wall motion analysis for detecting angiographically significant CAD through increased endocardial visualization at rest and during stress. 3,4,5,6

Although the primary goal of noninvasive testing in the assessment of suspected CAD is diagnosis, an important associated aim is to motivate at-risk patients to optimize their health behaviors ${ }^{7}$ for both primary and secondary prevention of CAD. The unequivocal detection and visualization of CAD may represent a "teachable moment" for patients and healthcare providers alike. ${ }^{8}$ Regrettably, limited evidence exists supporting the significance of the influence of noninvasive $\mathrm{CV}$ imaging on metrics of care in primary prevention settings. In particular, the impact of CSE on CV risk behaviors has not been demonstrated. We sought to evaluate the impact of CSE on CV risk behaviors in postmenopausal women presenting with symptoms and/or risk factors concerning for CAD.

This Stress Echocardiography in Menopausal Women at Risk for Coronary Artery Disease (SMART) substudy aimed to evaluate the prognostic value of CSE, coronary artery calcification, and cardiac biomarkers for prediction of $\mathrm{CV}$ events after 2 and 5 years in postmenopausal women experiencing chest pain symptoms or risk factors. ${ }^{9}$

\section{Materials and Methods}

\section{Study design}

This prospective multicenter study was approved by the Mayo Clinic Institutional Review Board and conducted at the three Mayo Clinic group-practice sites: Rochester, MN; Jacksonville, FL; and Scottsdale, AZ. The SMART trial ${ }^{9}$ enrolled women referred to the stress echocardiography laboratory for evaluation according to the eligibility criteria.

Peri- and postmenopausal women 40-65 years old and characterized by one of the following chest pain symptomatology groups were included: (1) asymptomatic and three or more risk factors $(\mathrm{RF})$ for $\mathrm{CAD}$; (2) atypical chest pain or exertional dyspnea and two or more RF for CAD; or (3) typical anginal chest pain with zero or one RF for CAD. Risk factors for $\mathrm{CAD}$ included being a current smoker or having quit smoking within the past 5 years; having diabetes (fasting glucose $\geq 120 \mathrm{mg} / \mathrm{dL}$, hemoglobin A1c [HbA1C] $>6.0$ ), or receiving medication; having a family history of premature $\mathrm{CAD}$ (defined as $\mathrm{CAD}$ in a female relative $<65$ years of age or a male relative $<55$ years of age) or hypertension (untreated systolic blood pressure [BP] $>140$ or diastolic BP $>90 \mathrm{~mm}$ $\mathrm{Hg}$ ); receiving antihypertensive medication, dyslipidemia (total cholesterol $>200 \mathrm{mg} / \mathrm{dL}$, or low-density lipoprotein [LDL] $>130 \mathrm{mg} / \mathrm{dL}$, or high-density lipoprotein [HDL]
$<35 \mathrm{mg} / \mathrm{dL}$ ), or receiving medication for lipid control; and being obese, defined as body mass index (BMI) $\geq 30 \mathrm{~kg} / \mathrm{m}^{2}$.

Perimenopausal status was defined as the absence or irregularity of menstrual periods for 6-12 months; postmenopausal status was defined as $>12$ months since patient's last menses. Surgical menopause included females who had prior bilateral salpingo-oophorectomy with or without hysterectomy. Patients were excluded if they had previous confirmation of CAD, documented left ventricular ejection fraction $(\mathrm{LVEF})<50 \%$, acute coronary syndrome, contraindication to exercise and/or pharmacologic stress testing, or known contraindications to contrast agent.

\section{Study procedures}

Contrast stress echocardiography. All women underwent CSE for left ventricular opacification (LVO) using the perfluorocarbon ultrasound contrast agent (Definity ${ }^{\circledR}$, Lantheus Medical Imaging, Billerica, MA) regardless of the baseline image quality, in order to provide optimal endocardial definition. One vial of Definity® was diluted with $0.9 \%$ saline (total volume $10 \mathrm{cc}$ ) and given as boluses of $0.5-1.0 \mathrm{~mL}$, followed by slow 3-5 mL saline flush. Exercise or dobutamine-CSE was performed according to standard protocols as previously described. ${ }^{9}$

Imaging was performed on commercially available ultrasound systems: iE33 (Philips Medical Systems, Bothell, WA), Sequoia 512 (Siemens Acuson, Mountain View, CA), or Vivid 7 (GE Healthcare, Princeton, NJ). Prior to contrast injection, baseline echocardiographic images were obtained from parasternal and apical windows. LVO images were acquired using low mechanical index (0.2-0.3) contrastspecific imaging using vendor presets in the apical 4-, 2-, and 3 -chamber and apical short-axis views at all stages of dobutamine-CSE and at rest and immediate postexercise-CSE stages. Machine settings were adjusted to optimize LVO images. All studies were recorded on videotape and digitized for storage. A 12-lead ECG was monitored continuously, and $\mathrm{BP}$ was obtained at each stage of CSE until heart rate recovery was achieved. Monitoring and electronic documentation of adverse events were performed.

Echocardiographic studies were analyzed off-line by two blinded readers and a third for consensus as necessary. Rest and stress images were reviewed side by side utilizing a 16segment model according to established criteria. ${ }^{10}$ LVEF was calculated using two-dimensional biplane volumes. Wall motion was evaluated using previously established criteria, ${ }^{10}$ and rest- stress wall motion score indexes (WMSIs) were calculated; briefly, segmental scores were categorized as follows: normal or hyperkinesis $=1$, hypokinesis $=2$, akinesis $=3$, dyskinesis $=4$, and aneurysmal $=5$. WMSI was derived as the sum of all scores, divided by the number of segments visualized. ${ }^{10}$ A normal WMSI is equal to 1.

Abnormal CSE was defined as the development of new or worsening of existing wall motion abnormalities during peak stress. This definition facilitates the identification of myocardial ischemia whereby segments that are normal or hypokinetic at rest demonstrate either a stress-induced improvement or a reduction in wall thickening, with interpretation as a normal versus ischemic segment response, respectively.

Quesionnaire. All subjects were invited to complete the Women's Heart Clinic Risk Assessment Questionnaire 
(WHCRAQ) at baseline (at time of CSE) and 2 years later (via US Postal Service). This survey of women's cardiovascular health assesses demographics, medical history, hormone therapy (HT), and CV risk behavior (smoking, exercise, and dietary fat and alcohol intake) parameters. Smoking status was evaluated by assessing cigarette smoking habits (never smoked, used to smoke, or current smoker) and the number of cigarettes consumed per day (zero through more than nine per day). Physical activity or exercise level was evaluated by reporting the total minutes exercised per week. Dietary fat intake was defined as "higher" if one or more servings of the following food items were consumed daily: chips, fried foods, fast foods, red meats, cheese made from cream or whole milk, egg yolks, or combination dishes made from these foods. Alcohol intake was evaluated by reporting the total number of alcoholic drinks per week. Current HT was considered if the subject was taking menopausal hormone therapy including estrogen or progesterone therapy as follows: estradiol (oral and transdermal); conjugated equine estrogen (Premarin, Wyeth Pharmaceuticals Inc., a subsidiary of Pfizer Inc., Philadelphia, PA), alone or combined with medroxyprogesterone acetate (Prempro, Wyeth Pharmaceuticals Inc., a subsidiary of Pfizer Inc., Philadelphia, PA); and natural progesterone (Prometrium, AbbVie Inc., North Chicago, IL).

CSE results were reviewed by the requesting clinician and recommendations made according to the patient's care team. Final analysis included all patients for whom there were baseline and 2-year follow-up data, excluding those patients who had undergone coronary angiography in the interim.

Statistical analysis of baseline data. After collecting the baseline data, we evaluated the relationship between each of the clinical cardiac risk behaviors and the result of the CSE (normal vs. abnormal). Clinical characteristics of patients were reported as numbers and proportions for categorical variables and mean (with standard deviation) for continuous variables with normal distribution. Comparison between groups with abnormal or normal CSE was performed using a two-sided Fisher's exact test. Paired $t$-test or nonparametric Wilcoxon paired sample testing was used for assessment of within-subject changes (baseline and 2-year follow-up). Bonferroni-corrected $p$-value ( $p=0.05 / 3)$ post hoc independent and related samples $t$-tests were employed to test for differences between group and over time, respectively. All analyses were performed using JMP version 9.1 (SAS Institute Inc., Cary, NC). To evaluate reliability of CSE, interobserver agreement was assessed in 43 randomly assigned patients by two independent observers; intraobserver variability was assessed on two occasions at least 5 months apart. Agreement was reported using kappa coefficient.

\section{Results}

From January 2004 to August 2007, a total of 377 women completed the study ( $7.4 \%$ perimenopausal, $52.5 \%$ postmenopausal, and $46.2 \%$ with surgical menopause). Framingham risk score (FRS) for CAD was $6.51 \pm 4.4 \%$ (median $6 \%$, range: 1-27\%). Symptoms (atypical chest pain or exertional dyspnea) and two or more RF for $\mathrm{CAD}$ were present in $63.1 \%$; $34 \%$ were asymptomatic with three or more RF for $\mathrm{CAD}$; and $2.2 \%$ of the women had typical anginal chest pain with zero or one RF for CAD. In addition, 11 women terminated the study early (4 with adverse events [back pain at time of contrast], 4 with consent withdrawal, and 3 with other causes). Thus, $366(97.1 \%)$ women (mean age $54.4 \pm 5.5$ years; range 40-65, BMI 31.4 \pm 6.68 , Caucasian in 95\%) completed CSE and were invited to participate in the WHCRAQ survey. Clinical characteristics of the participant population are shown in Table 1. Of the 366 women, 203 (55\%) completed both baseline and 2-year follow-up surveys. Twenty-nine of the 203 women reported having a coronary angiography (CA) and hence were excluded from final analysis (to avoid confounding the effect of other diagnostic testing). Thus, 174 women were included in the final analysis (follow-up duration $719 \pm 78$ days).

Patient characteristics and lifestyle/hormone therapy results of the respondent population are shown in Table 2. CSE was interpretable in all 174 women ( $81 \%$ of women performed exercise-CSE and 19\% dobutamine-CSE). Mean LVEF was $61 \% \pm 6 \%$ at rest; $70 \% \pm 7 \%$ at peak stress $(p<0.001)$. CSE was abnormal in 17 women $(9.8 \%)$, with

\section{Table 1. Clinical Characteristics of Study} Population $(N=366)$

\begin{tabular}{|c|c|}
\hline Variable & Overall $(\mathrm{n}=366$ \\
\hline Age, years & $54.4 \pm 5.24$ \\
\hline $\mathrm{BMI}, \mathrm{kg} / \mathrm{m}^{2}$ & $31.4 \pm 6.68$ \\
\hline \multicolumn{2}{|l|}{$\mathrm{CSE}, n(\%)$} \\
\hline Exercise & $278(75.96)$ \\
\hline Dobutamine & $88(24.04)$ \\
\hline Caucasian, $n(\%)$ & $349(95.4)$ \\
\hline \multicolumn{2}{|l|}{ CAD risk factors, $n(\%)$} \\
\hline Smoking history $^{\mathrm{a}}$ & $92(25.14)$ \\
\hline Diabetes mellitus & $56(15.3)$ \\
\hline Family history CAD & $222(60.7)$ \\
\hline Hypertension & $231(63.1)$ \\
\hline Dyslipidemia & $292(79.8)$ \\
\hline $\mathrm{BMI} \geq 30 \mathrm{~kg} / \mathrm{m}^{2}$ & $205(56.0)$ \\
\hline Hormone therapy ${ }^{\mathrm{b}}$ & $103(28.1)$ \\
\hline \multicolumn{2}{|l|}{ Chest pain groups, $n(\%)$} \\
\hline Asymptomatic & $125(34.2)$ \\
\hline Atypical chest pain & $233(63.7)$ \\
\hline Typical chest pain & $8(2.2)$ \\
\hline \multicolumn{2}{|l|}{ Medications, $n(\%)$} \\
\hline ACE inhibitor & $64(17.5)$ \\
\hline Alpha blocker & $5(1.37)$ \\
\hline Beta blocker & $89(24.3)$ \\
\hline Antiplatelet/ASA & $110(30.1)$ \\
\hline Anticoagulant & $10(2.7)$ \\
\hline A-II receptor blocker & $49(13.4)$ \\
\hline Calcium channel blocker & $31(8.5)$ \\
\hline Lipid-lowering agent & $147(40.2)$ \\
\hline
\end{tabular}

Continuous variables are presented as mean $\pm \mathrm{SD}$; categorical variables are presented as number and percentage.

${ }^{\mathrm{a}}$ Current or ex-smoker.

${ }^{b}$ Mean \pm SD years of hormone therapy use was $2.74 \pm 5.04$ years. Types of hormone therapy included estradiol (oral and transdermal); conjugated equine estrogen (Premarin, Wyeth Pharmaceuticals Inc., a subsidiary of Pfizer Inc., Philadelphia, PA), alone or combined with medroxyprogesterone acetate (Prempro, Wyeth Pharmaceuticals Inc., a subsidiary of Pfizer Inc., Philadelphia, PA); and natural progesterone (Prometrium, AbbVie Inc., North Chicago, IL).

ACE, angiotensin converting enzyme; ASA, acetylsalicylic acid; BMI, body mass index; CAD, coronary artery disease; CSE, contrast stress echocardiography; HR, heart rate; SD, standard deviation. 
Table 2. Clinical Characteristics and Cardiovascular Risk BeHaViors and Hormone Therapy Use IN WOMEN RESPONDING to THE Women's Heart Clinic Risk Assessment QuestionNAIRE AT BASELINE (TIME OF CSE), Grouped by CSE Results $(N=174)$

\begin{tabular}{lccc}
\hline & $\begin{array}{c}\text { Abnormal } \\
\text { CSE } \\
(\mathrm{n}=17)\end{array}$ & $\begin{array}{c}\text { Normal } \\
\text { CSE } \\
(\mathrm{n}=157)\end{array}$ & p-value \\
\hline Age & $63 \pm 5$ & $61 \pm 5$ & 0.22 \\
BMI $\left(\mathrm{kg} / \mathrm{m}^{2}\right)$ & $34 \pm 8$ & $33 \pm 9$ & 0.46 \\
Hypertension & $12(71 \%)$ & $83(53 \%)$ & 0.45 \\
Diabetes & $4(24 \%)$ & $13(8 \%)$ & 0.04 \\
Hyperlipidemia & $13(76 \%)$ & $120(78 \%)$ & 0.91 \\
Smoking history & & & \\
$\quad$ Nonsmokers & $10(59 \%)$ & $75(48 \%)$ & 0.03 \\
$\quad$ Current smokers & $4(24 \%)$ & $13(8 \%)$ & \\
$\quad$ Ex-smokers & $3(17 \%)$ & $69(44 \%)$ & \\
Alcohol intake & $3(17 \%)$ & $60(38 \%)$ & 0.10 \\
Exercise & $13(76 \%)$ & $93(59 \%)$ & 0.22 \\
Higher-fat diet & $13(76 \%)$ & $145(92 \%)$ & 0.03 \\
Current hormone therapy & $2(12 \%)$ & $42(27 \%)$ & 0.19 \\
Number of cigarettes & $0.35 \pm 1.4$ & $1.42 \pm 5.1$ & 0.051 \\
$\quad$ per day & & & \\
Number of alcohol & $1.94 \pm 5.2$ & $2.5 \pm 3.5$ & 0.031 \\
$\quad$ drinks per week & & & \\
Exercise minutes & $50.5 \pm 46$ & $37 \pm 46$ & 0.264 \\
$\quad$ per week & & & \\
\hline
\end{tabular}

Continuous variables are presented as mean $\pm \mathrm{SD}$; categorical variables are presented as number and percentage.

${ }^{\mathrm{a}}$ Higher-fat diet if intake is one or more times per day.

mean peak WMSI $=1.37 \pm 0.263$. Total agreement was $88 \%$ $(\kappa, 0.685[0.13])$ for intraobserver and $87 \%(\kappa, 0.754[0.16])$ for interobserver CSE wall motion assessment.

In the abnormal-CSE group of women, the following changes in CV risk behaviors over 2 years were observed: (1) a reduction of total number of alcohol drinks per week, (2) a reduction in HT use, (3) an increase in total exercise time (minutes per week), (4) an increased higher-fat diet, and (5) a decrease in the number of cigarettes smoked per day (4 of 17 women were smokers at baseline but had stopped smoking at the 2-year follow-up). However, the only statistically significant change observed was for smoking cessation (across the only smokers in the abnormal-CSE group, a mean difference of -1.5 cigarettes per day, $p=0.014$ ) (Table 3 ).

Similarly, in the normal-CSE group, the following changes in $\mathrm{CV}$ risk behaviors over 2 years were observed: (1) a decrease in the number of cigarettes smoked per day, despite an increased number of women smokers at 2-year follow-up (13 of 157 were smokers at baseline of whom 5 stopped smoking, but an additional 6 nonsmokers became smokers to total 14 smokers at 2-year follow-up); (2) a decrease in the number of women on HT; (3) an increase in the total exercise time (minutes per week); (4) an increase in the number of women with a higher-fat diet; and (5) an increase in the total number of alcohol drinks per week. However, the only statistically significant change was observed in the number of alcohol drinks per week (across the entire normal-CSE group, a mean difference of $+0.38, p=0.009)$ (Table 3 ).

\section{Discussion}

To our knowledge, this is the largest prospective cohort study in postmenopausal women at low to intermediate risk for CAD to address the question of CSE diagnosis-dependent effects on cardiac-related lifestyle factors. Our study illustrated that women may cognitively modify (even if modestly) their perceptions of receiving (1) an unfavorable diagnosis (abnormal CSE in this study) in a positive direction (positive lifestyle modifications) as we observed with a significant reduction in smoking or (2) a favorable/reassuring (normal CSE in this study) diagnosis in a negative direction (less attention to modification of $\mathrm{CV}$ risk behaviors) as we demonstrated with the significant increase in alcohol intake. We

Table 3. Cardiac Lifestyle and Hormone Therapy Use in Women Responding to the WHCRAQ Survey at Baseline (Time of CSE) and 2-Year Follow-Up, Grouped by CSE Results $(N=174)$

\begin{tabular}{|c|c|c|c|c|c|c|c|}
\hline & \multicolumn{3}{|c|}{ Abnormal CSE $(\mathrm{n}=17)$} & \multicolumn{3}{|c|}{ Normal CSE $(\mathrm{n}=157)$} & \multirow{2}{*}{$\begin{array}{l}\text { p-value (comparing } \\
\text { abnormal vs. normal } \\
\text { CSE at } 2 \text { years) }\end{array}$} \\
\hline & Baseline & 2 years & $\mathrm{p}-$ value $^{\mathrm{a}}$ & Baseline & 2 years & $\mathrm{p}-$ value $^{\mathrm{a}}$ & \\
\hline $\begin{array}{l}\text { Mean number of cigarettes } \\
\text { per day in smokers }\end{array}$ & $1.5 \pm 0.58$ & 0 & 0.014 & $17.2 \pm 7.8$ & $12.9 \pm 7.7$ & 0.162 & $<0.0001$ \\
\hline $\begin{array}{l}\text { Total number of drinks } \\
\text { per week }\end{array}$ & $1.94 \pm 5.2$ & $1.2 \pm 2.9$ & 0.449 & $2.5 \pm 3.5$ & $2.9 \pm 4.2$ & 0.009 & 0.05 \\
\hline $\begin{array}{l}\text { Total exercise minutes } \\
\text { per week }\end{array}$ & $50.5 \pm 46$ & $51.3 \pm 47$ & 0.839 & $37 \pm 46$ & $40 \pm 47$ & 0.410 & 0.34 \\
\hline Higher-fat diet ${ }^{\mathrm{c}}$ & $13(76 \%)$ & $15(88 \%)$ & 0.697 & $145(92 \%)$ & $149(94.9 \%)$ & 0.350 & 0.03 \\
\hline Hormone therapy ${ }^{\mathrm{d}}$ & $2(12 \%)$ & $1(5.9 \%)$ & 0.562 & $42(27 \%)$ & $31(19.7 \%)$ & 0.14 & 0.11 \\
\hline
\end{tabular}

Continuous variables are presented as mean $\pm \mathrm{SD}$; categorical variables are presented as number and percentage.

${ }^{a}$ Bonferroni-corrected significant $p$-value $<(p=0.05 / 3)$.

${ }^{b}$ In the abnormal-CSE group, 4 of 17 were smokers at baseline and had stopped smoking at the 2-year follow-up; in the normal-CSE group, 13 of 157 were smokers at baseline, and 5 had stopped smoking at the 2-year follow-up, but an additional 6 nonsmokers became smokers, for a total of 14 smokers at the 2-year follow-up.

${ }^{c}$ Higher-fat diet if intake is one or more times per day.

${ }^{\mathrm{d}}$ In the abnormal-CSE group, 2 were taking hormone therapy (both containing progesterone) at baseline; 1 was no longer taking hormone therapy at the 2-year follow-up. In the normal-CSE group, 6 of 42 women were taking progesterone. At the 2-year follow-up, 1 of 6 had discontinued progesterone, and 1 had newly started taking progesterone, so there was no overall change in the number taking progesterone. 
also demonstrated other $\mathrm{CV}$ risk behavior changes (positive or negative) in both normal- and abnormal-CSE groups that may have shown diagnosis-related characteristics but were not of statistical significance.

Our study complements other reports demonstrating the influence that imaging modalities can have on healthy behavioral change and, in particular, smoking cessation. ${ }^{11,12}$ The expanded growth in the use of CV imaging suggests a need for research focused on the impact of noninvasive $\mathrm{CV}$ testing and influence on clinical care and prognosis. Through multiple pathways, CV noninvasive imaging tests may potentially promote lifestyle modification. However, evidence is lacking that such testing may result in better health outcomes. This finding is even more important considering that therapeutic lifestyle changes in conjunction with an aggressive multidrug regimen targeted toward the normalization of the major $\mathrm{CV}$ risk factors will neutralize the atherogenic milieu and markedly decrease the risk of adverse CV events and need for revascularization procedures. ${ }^{13}$

The meta-analysis by Hackman et al. ${ }^{8}$ included randomized clinical trials that compared primary prevention patients undergoing noninvasive CV imaging versus control groups receiving usual or standard care. These trials evaluated the impact of using noninvasive imaging modalities, including echocardiography, in asymptomatic middle-aged patients with no history of $\mathrm{CV}$ diseases to that upon patients with major $\mathrm{CV}$ risk factors in regard to lifestyle modifications (smoking cessation in four trials ${ }^{11,14,15}$ dietary modification in one trial, ${ }^{14}$ or physical activity in one trial ${ }^{15}$ ). The overall results of this meta-analysis failed to show a statistically significant effect of noninvasive imaging on smoking cessation, dietary improvement, or physical activity in patients without known CAD. Our observations were supportive of these prior studies, ${ }^{11,14,15}$ showing only a modest nonsignificant impact on physical activities and no impact on dietary change. However, we found that smoking cessation was positively impacted by CSE results. The emphasis on smoking cessation and addressing nicotine dependence in women is critical, since the risk of death from cigarette smoking continues to increase among women, and the $\mathrm{CV}$ risks for women are at least twofold that for men when compared with persons who have never smoked. ${ }^{16}$

The relevance of our findings is further reinforced if we consider that in one large national survey of women contacted through random digit dialing, women reported having taken numerous preventive actions, whereas only $29 \%$ reported quitting smoking ( $25 \%$ white vs. $38 \%$ nonwhite). ${ }^{17}$

At the same time, our data showed that CSE results failed to significantly impact many other $\mathrm{CV}$ risk behaviors. Although the findings related to exercise intentions and increase in total exercise duration per week were not statistically significant in either group (normal or abnormal CSE), it is still encouraging and is in agreement with prior research involving the benefit of advanced cardiac imaging on behavioral measures, including physical activity. ${ }^{15}$

Paradoxically, in our current study, a higher-fat diet was reported in both CSE groups, and alcohol consumption increased in the normal versus abnormal CSE testing. Although we cannot explain the increased higher-fat diet in the abnormal-CSE group, we can argue that the absence of demonstrated pathologic findings in CSE testing may reassure patients with risk factors but no manifest detectable disease. ${ }^{8}$
However, it can also be argued that a modest increment in alcohol consumption is a desirable CV risk-reduction intervention; indeed, the alcohol level reported in our study cohort both at baseline and at 2-year follow-up was still well within the recommended limits for women.

The present study confirms that it is difficult to achieve risk-factor control in routine clinical practice even after a positive CSE in postmenopausal women. ${ }^{18}$ In general, impediments to implementation of guidelines include time pressures, lack of organizational support, and patient resistance to behavioral change. In particular, barriers hindering adherence to $\mathrm{CV}$ disease-prevention recommendations are common among women and include family and caretaking responsibilities, stress, sleep deprivation, fatigue, and lack of personal time. ${ }^{19}$ However, these variables were not directly assessed in our current study.

Our study has a particular relevance to clinical practice. Physicians are encouraged to convey a persuasive message addressing the various CV risk behaviors, and this is best achieved or emphasized around the time of the diagnostic test-result discussion. The most robust interventions are multifaceted, are interactive, and incorporate decision systems and feedback. ${ }^{20}$

The major strength of the study is the clinical "real-world" setting in which it was conducted, the large number of patients studied prospectively, and the uniqueness of the sampled population (low- to intermediate-risk postmenopausal women, a group that has recently been identified as having an increasing incidence of CV disease). However, some limitations need to be addressed. The first limitation concerns the self-reporting of behavioral variables (with a possibility of underreporting or overreporting of undesirable and desirable lifestyle behaviors, respectively). However, we obtained a longer follow-up ( 2 years), which is crucial to assess the healthy patterns of diet and exercise, both being central to the prevention of heart diseases. Second, it was not possible to isolate the potential contributing factors that may have influenced the overall changes in health behaviors, specifically with respect to smoking cessation, and no causal relationship between the CSE results and the behavioral changes can be ensured. Third, we included all types of HT in the WHCRAQ, including progesterone preparations, which are characteristically administered to women seeking HT but without history of prior hysterectomy. Subgroup analysis was not feasible, owing to the small number of women reporting HT and the even smaller number reporting progesterone preparation use. However, recent information regarding the "null effect" of combined HT (with both estrogen and progesterone) administered early (within 4 years) of menopausal onset suggests that there is no untoward effect upon $\mathrm{CV}$ surrogates (coronary artery calcification and/or carotid intima media thickness). ${ }^{21}$ Fourth, with respect to dietary details in the WHCRAQ, we unfortunately did not specify "good" (unsaturated) versus "bad" (saturated) fats; nor did we quantify the exact amount of alcohol consumed.

Despite these limitations, we think it is very intriguing to contemplate that the results of a diagnostic test may influence patient lifestyle. Thus, our study is uniquely hypothesisgenerating and sex-specific (to women). We were limited by the post hoc subgroup analysis of the larger prospective SMART trial. The lack of significance in other CV riskbehaviors changes may reflect a small effect size, low power, 
and/or low response rate (55\%). Nevertheless, we believe that the strictly defined criteria of $\mathrm{CV}$ risk behaviors in the WHCRAQ and the long-term follow-up allowing for consistent behavioral assessment may reduce this limitation. We have evaluated only the noninvasive imaging modality CSE as part of the SMART trial; hence, our results may not be generalizable to other noninvasive imaging modalities in women.

Further, we didn't evaluate the diagnosis-dependent effects on $\mathrm{CV}$ medication adherence. Other limitations include the incomplete response rate $(55 \%)$ of this survey study and the use of an internal clinic questionnaire that did not clearly differentiate "good" and "bad" dietary fats rather than a validated dietary questionnaire. Nonetheless, this work suggests future directions for research into the effect of noninvasive CV test results and potential impact on modification of $\mathrm{CV}$ risk through enhanced lifestyle counseling and adherence to hearthealthy behaviors.

\section{Conclusions}

In a substudy of the SMART trial assessing cardiac lifestyle behavioral changes, smoking cessation was positively impacted by CSE testing results at 2-year follow-up. However, a paradoxically higher-fat diet was reported in both CSE groups (normal and abnormal), and higher alcohol consumption was reported more often in those with normalversus abnormal-CSE testing.

These findings suggest the intriguing possibility that in postmenopausal women, $\mathrm{CV}$ health behaviors may be altered by the results of stress testing. Specifically, the possibility of a positive impact of CSE testing results on the commitment of smoking postmenopausal women to smoking cessation is suggested but remains uncertain. Future research is necessary to explore the potential impact of noninvasive $\mathrm{CV}$ imaging studies upon lifestyle change and primary prevention in women, beyond the immediate diagnostic implications of the test result.

\section{Acknowledgments}

The SMART trial (clinicalTrial.gov identifier\# NCT00162370) was funded solely by Lantheus Medical Imaging (North Billerica, MA). Lantheus Medical Imaging provided the contrast agent (Definity) and additional research funding under grant \#DMP 115-407.

\section{Author Disclosure Statement}

Sharon L. Mulvagh has received research grants from Lantheus Medical Imaging and Astellas Pharma Inc. She has been a member of the Advisory Board for GE HealthCare. For all other authors, no conflicts of interest exist.

\section{References}

1. Lindquist R, Boucher JL, Grey EZ, et al. Eliminating untimely deaths of women from heart disease: Highlights from the Minnesota Women's Heart Summit. Am Heart J 2012;163:39-48,e31.

2. Mieres JH, Shaw LJ, Arai A, et al. Role of noninvasive testing in the clinical evaluation of women with suspected coronary artery disease: Consensus statement from the Cardiac Imaging Committee, Council on Clinical Cardiol- ogy, and the Cardiovascular Imaging and Intervention Committee, Council on Cardiovascular Radiology and Intervention, American Heart Association. Circulation 2005; 111:682-696.

3. Plana JC, Mikati IA, Dokainish H, et al. A randomized crossover study for evaluation of the effect of image optimization with contrast on the diagnostic accuracy of dobutamine echocardiography in coronary artery disease: The OPTIMIZE Trial. JACC Cardiovasc Imaging 2008;1:145-152.

4. Rainbird AJ, Mulvagh SL, Oh JK, et al. Contrast dobutamine stress echocardiography: Clinical practice assessment in 300 consecutive patients. J Am Soc Echocardiogr 2001;14:378-385.

5. Abdelmoneim SS, Bernier M, Scott CG, et al. Safety of contrast agent use during stress echocardiography: A 4-year experience from a single-center cohort study of 26,774 patients. JACC Cardiovasc Imaging 2009;2:1048-1056.

6. Dolan MS, Gala SS, Dodla S, et al. Safety and efficacy of commercially available ultrasound contrast agents for rest and stress echocardiography a multicenter experience. J Am Coll Cardiol 2009;53:32-38.

7. O'Malley PG. The proof of atherosclerosis imaging is in the evidence: Where are the studies? Arch Intern Med 2011;171:976.

8. Hackam DG, Shojania KG, Spence JD, et al. Influence of noninvasive cardiovascular imaging in primary prevention: Systematic review and meta-analysis of randomized trials. Arch Intern Med 2011;171:977-982.

9. Abdelmoneim SS, Bernier M, Hagen ME, et al. A multicenter, prospective study to evaluate the use of contrast stress echocardiography in early menopausal women at risk for coronary artery disease: Trial design and baseline findings. J Womens Health (Larchmt) 2013;22:173-183.

10. Lang RM, Bierig M, Devereux RB, et al. Recommendations for chamber quantification: A report from the American Society of Echocardiography's Guidelines and Standards Committee and the Chamber Quantification Writing Group, developed in conjunction with the European Association of Echocardiography, a branch of the European Society of Cardiology. J Am Soc Echocardiogr 2005;18:1440-1463.

11. Bovet P, Perret F, Cornuz J, Quilindo J, Paccaud F Improved smoking cessation in smokers given ultrasound photographs of their own atherosclerotic plaques. Prev Med 2002;34:215-220.

12. Rodondi N, Auer R, Devine PJ, O'Malley PG, Hayoz D, Cornuz J. The impact of carotid plaque screening on motivation for smoking cessation. Nicotine Tob Res 2008; 10:541-546.

13. O'Keefe JH, Carter MD, Lavie CJ. Primary and secondary prevention of cardiovascular diseases: A practical evidence-based approach. Mayo Clin Proc 2009;84:741-757.

14. Lederman J, Ballard J, Njike VY, Margolies L, Katz DL. Information given to postmenopausal women on coronary computed tomography may influence cardiac risk reduction efforts. J Clin Epidemiol 2007;60:389-396.

15. O'Malley PG, Feuerstein IM, Taylor AJ. Impact of electron beam tomography, with or without case management, on motivation, behavioral change, and cardiovascular risk profile: A randomized controlled trial. JAMA 2003;289: 2215-2223.

16. Thun MJ, Carter BD, Feskanich D, et al. 50-year trends in smoking-related mortality in the United States. N Engl J Med 2013;368:351-364. 
17. Mosca L, Mochari-Greenberger H, Dolor RJ, Newby LK, Robb KJ. Twelve-year follow-up of American women's awareness of cardiovascular disease risk and barriers to heart health. Circ Cardiovasc Qual Outcomes 2010;3:120-127.

18. Bhatt DL, Steg PG, Ohman EM, et al. International prevalence, recognition, and treatment of cardiovascular risk factors in outpatients with atherothrombosis. JAMA 2006;295:180-189.

19. Mosca L, Benjamin EJ, Berra K, et al. Effectiveness-based guidelines for the prevention of cardiovascular disease in women-2011 update: A guideline from the American Heart Association. J Am Coll Cardiol 2011;57:1404-1423.

20. Farkouh ME, Boden WE, Bittner V, et al. Risk factor control for coronary artery disease secondary prevention in large randomized trials. J Am Coll Cardiol 2013;61:1607-1615.
21. Kronos Early Estrogen Prevention Study (KEEPS) study results. The North American Menopause Society (NAMS) 23rd annual meeting, 2012, in Orlando, FL. Available at: http://www.menopause.org/annual-meetings/2012-meeting/ keeps-report (accessed on March 30, 2014).

Address correspondence to: Sharon L. Mulvagh, MD Division of Cardiovascular Medicine Mayo Clinic 200 First Street $S W$ Rochester, MN 55905

E-mail: smulvagh@mayo.edu 